\title{
Exact Dynamic Modeling of PWM DC-to-DC Power Converters-Part I: Continuous Conduction Mode
}

\author{
Justin Simmons, Richard Tymerski \\ Maseeh College of Engineering and Computer Science, Portland State University, Portland, USA \\ Email: tymerski@ee.pdx.edu
}

How to cite this paper: Simmons, J. and Tymerski, R. (2021) Exact Dynamic Modeling of PWM DC-to-DC Power Converters-Part I: Continuous Conduction Mode. Journal of Power and Energy Engineering, 9, 26-47.

https://doi.org/10.4236/jpee.2021.95002

Received: October 14, 2020

Accepted: May 19, 2021

Published: May 22, 2021

Copyright $\odot 2021$ by author(s) and Scientific Research Publishing Inc. This work is licensed under the Creative Commons Attribution International License (CC BY 4.0).

http://creativecommons.org/licenses/by/4.0/

\begin{abstract}
A general approach is presented by which the exact frequency response of any transfer function of switched linear networks can be determined. This is achieved with a describing function approach using a state space equation formulation. This work presents a somewhat simplified set of equations to one previously given by one of the authors. To demonstrate application of the general formulation, the frequency responses of switched networks used as PWM DC-to-DC converters operating in continuous conduction mode (CCM) under voltage mode control are derived. (The accompanying paper, Part II, will present results for converters operating in discontinuous conduction mode (DCM)). From the general sets of equations developed here, both the control to output and input source variation to output frequency responses are derived. The describing function approach enables exact frequency response determination, even at high frequencies where the accuracy using average models may be compromised. Confirmation of the accuracy of the derived models is provided by comparing the responses with those obtained using the commercial simulator PSIM on a PWM boost converter. The magnitude and phase responses are shown to match perfectly over the full range of frequencies up to close to half the switching frequency. Matlab code that implements the models is given such that the user can easily adapt for use with other PWM converter topologies.
\end{abstract}

\section{Keywords}

Describing Function, DC-to-DC Switching Power Converter, Small-Signal Modeling, Frequency Response

\section{Introduction}

DC-to-DC converters are an important and widely used class of power 
processing systems. The input DC voltage is transformed to a higher or lower level, whilst achieving high power efficiency, that is, minimizing power losses. To achieve this, the controlling element is usually a set of switches. Typically these systems require tight control of the output voltage level despite variation in the input voltage and/or load current. Consequently negative feedback control is invariably used to achieve this requirement. To obtain an effective feedback design, an accurate determination of certain transfer functions need to be made. Generally small-signal averaged models are used for this purpose. In this work, however, describing function models are derived. The models derived are closed form expressions which contain state matrices of the switched networks along with the length of the various subintervals. In this paper, more specifically models for converters operating in continuous conduction mode (CCM) are considered.

The describing function modeling method is used for non-linear systems by providing a sinusoidal input and then determining the magnitude and phase at this frequency component in the output spectrum. The use of average models, widely used for this purpose, generally fail to accurately predict the response at high frequencies. Describing function modeling methods have become more popular in recent years as greater accuracy at high frequencies is sought after [1] [2] [3].

The purpose of this paper is 1) to review a describing function modeling approach previously discussed by the author [4] [5] [6], and 2) to further develop this modeling approach. This has resulted in a simplification in the model derivation. Next, 3) we demonstrate the derivation of exact frequency response models for PWM converters operating in CCM under voltage mode control using this newer formulation, and furthermore, 4) Matlab code is provided which may be adapted to determine exact frequency responses for any PWM converter topology. In this paper, confirmation of the accuracy of this modeling method is also provided by comparison with results obtained from a commercial simulator. With the latest developments included, this paper comprises an up-to-date representation of this very effective modeling method. A further purpose of this work is that with the derivation of the complete set of transfer functions to determine PWM converter frequency responses, in future work, these will be used to aid in the development of simplified, yet accurate, circuit models.

Since we are considering time varying networks, it is natural to consider the time varying transfer function [7] from which the required frequency response could be derived. In Section 4, starting with the definition of the time varying transfer function an expression is derived for the frequency response of the frequency component in the output spectrum that is being sought after, i.e. the same frequency as that of the input perturbational sine wave. This expression contains small signal state, subinterval time and input source perturbational terms. These terms are dealt with by deriving a general small signal difference equation for any switching subinterval. This is undertaken in Section 3.

The layout of the paper is as follows. The next section, Section 2, a quick review of the structure of closed loop DC-to-DC switching regulators is given. The 
subsequent section, as previously mentioned, serves to develop a mathematical representation of the system using state-space analysis. Starting with the large signal model, both DC and AC small signal discrete time models are derived. A general expression for the zero order component of the time varying transfer function, $H_{0}(j \omega)$, is then be developed in Section 4. In this way, any transfer function of interest can be derived. Specifically, we will look at the control input to output voltage transfer function, $\frac{\hat{v}_{\text {out }}}{\hat{r}}$, and input source to output voltage transfer function, $\frac{\hat{v}_{\text {out }}}{\hat{v}_{g}}$. In Section 5 , the method will be applied to a boost PWM converter operating in continuous conduction mode. Confirmation of the derived models is presented in Section 6, by comparison with frequency responses obtained with a commercial simulator. Finally, the Conclusion (Section 7) summarizes the main results obtained.

\section{DC-to-DC Switching Voltage Regulators}

Figure 1 shows a representation of a DC-to-DC switching power regulator operating in voltage mode (VM). VM is used to describe a converter whose sawtooth signal is externally supplied rather than generated by some output of the system. Control is achieved by duty ratio $d$ variation which can be adjusted to maintain an average (DC) output voltage $V_{\text {out }}(t)$ despite variations of the supply voltage $v_{g}(t)$. The power stages of switched converters consist of only storage elements and switches making the power conversion theoretically lossless with ideal components. They are essential for portable devices whose supply voltage will lower as batteries are drained and for integrated circuits whose integrity would be severely compromised by heat dissipation. In Figure 1, the output voltage $v_{\text {out }}(t)$ across the load resistance $R_{l}$ is sensed through a gain

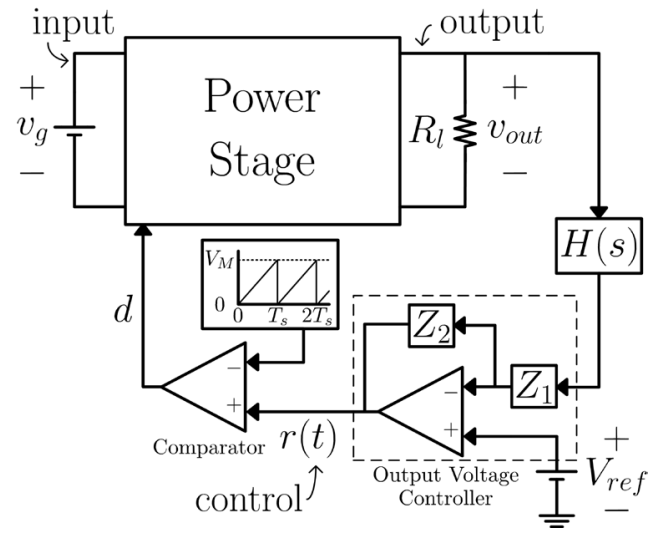

Figure 1. General diagram of an VM DC-to-DC power converter featuring an output voltage controller. The output voltage is sensed through $H(s)$ and fed through a compensator to produce a signal $r(t)$, which is then used by a comparator along with an externally provided sawtooth signal to produce a rectangular wave with period $T_{s}$ and duty ratio $d$ that controls the active switch. The input $v_{g}(t)$ contains both DC $\left(V_{g}\right)$ and AC $\left(\hat{v}_{g}(t)\right)$ components. 
$H(s)$ and fed into the output voltage controller along with an external reference voltage $V_{\text {ref }}$. Impedances $Z_{1}$ and $Z_{2}$ determine the gain and the pole and zero locations and are made up of capacitors, resistors, or both depending on the particular compensator used in the output voltage controller. The compensator produces a control input signal $r(t)$ :

$$
r(t)=V_{\text {ref }}+\frac{Z_{2}}{Z_{1}}\left[V_{\text {ref }}-v_{\text {out }}(t) H(s)\right]
$$

The control input signal $r(t)$ is fed to a comparator that compares it to an externally introduced sawtooth signal of period $T_{s}$ and amplitude $V_{M}$, producing a rectangular wave with a duty ratio $d$ and switching period $T_{s}$ as illustrated in Figure 2 that regulates the switching between topologies of the power stage. Adjusting $H(s)$ appropriately so that $V_{\text {ref }}=V_{\text {out }}(t) H(s)$, where $V_{\text {out }}(t)$ is the DC component of $v_{\text {out }}(t)$, makes:

$$
R=V_{\text {ref }}
$$

where $R$ is the constant large signal (DC) component of $r(t) . D$, the DC component of $d$, is then determined by:

$$
D=\frac{V_{r e f}}{V_{M}}
$$

A good power converter will have minimal change in $v_{\text {out }}(t)$ with respect to change in $v_{g}(t)$, thus designing a power converter requires the satisfaction of a minimizing sensitivity of the output voltage to variations in the input voltage, or audiosusceptibility, represented by $\frac{\hat{v}_{\text {out }}}{\hat{v}_{g}}$ over a specified frequency range where $\hat{v}_{g}(t)$ and $\hat{v}_{\text {out }}(t)$ are the small signal (AC) components of the input $v_{g}(t)$ and $v_{\text {out }}(t)$, respectively. The design of a compensator requires the control-to-output transfer function $\frac{\hat{v}_{\text {out }}}{\hat{r}}$ where $\hat{r}$ is the AC component of the control input signal $r(t)$. The control design aims to provide an adequate level of positive phase margin.

\section{Large and Small Signal State Space Model}

State space analysis will be utilized to develop expressions that describe the topological states of the switching converters. The large signal model will first be

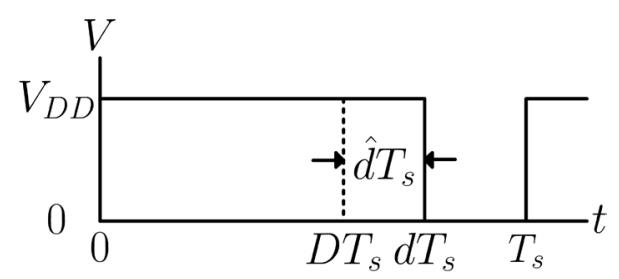

Figure 2. Rectangular wave produced by the comparator which regulates how long the active switch $Q$ is turned on. The time $d T_{s}$ is the actual time that the active switch is on in the presence of perturbations while $D T_{s}$ is the DC average time that the active switch is on in the absence of perturbations. 
developed and then perturbed to obtain the DC and AC the small signal models. While there is generally one switching device that controls the switching between two topologies, more topologies may exist should certain conditions be satisfied such as in Discontinuous Conduction Mode (DCM). Integer $N_{s}$ denotes the number of topologies that are present within a switching period $T_{s}$ The time spent in a particular topology will define a subinterval so that $T_{s}$ is divided into $N_{s}$ different subintervals. The general state space represention for a time varying system is:

$$
\begin{aligned}
& \dot{x}(t)=A(t) x(t)+B(t) u(t) \\
& y(t)=C^{\mathrm{T}}(t) x(t)+E(t) u(t)
\end{aligned}
$$

where $x(t)$ is the state vector consisting of both the large signal and small signal values of each inductor current and capacitor voltage, $\dot{x}(t)$ is its derivative, $y(t)$ is the output of the system, and $u(t)$ is the input vector. Without loss of generality, the input voltage, $v_{g}(t)$, is the only input considered here, thus making $u(t)$ a scalar:

$$
\begin{gathered}
x(t)=\left[\begin{array}{c}
i_{1}(t) \\
\vdots \\
i_{k}(t) \\
v_{1}(t) \\
\vdots \\
v_{l}(t)
\end{array}\right]=\left[\begin{array}{c}
I_{1}(t)+\hat{i}_{1}(t) \\
\vdots \\
I_{k}(t)+\hat{i}_{k}(t) \\
V_{1}(t)+\hat{v}_{1}(t) \\
\vdots \\
V_{l}(t)+\hat{v}_{l}(t)
\end{array}\right] \\
u(t)=U+\hat{u}(t)=v_{g}(t)=V_{g}+\hat{v}_{g}(t)
\end{gathered}
$$

for $k$ inductors and $l$ capacitors in the power stage of the converter. For a piecewise linear system the equations in (4) can be replaced by a set of $N_{s}$ linear time invariant (LTI) state equations of the form:

$$
\begin{aligned}
& \dot{x}(t)=A_{i} x(t)+B_{i} u(t) \\
& y(t)=C_{i}^{\mathrm{T}} x(t)+E_{i} u(t)
\end{aligned}
$$

for $t_{i} \leq t \leq t_{i+1}, i \in\left(1,2, \cdots, N_{s}\right)$, where $A_{i}$ and $B_{i}$ are the system and input matrices, respectively, that find the state derivative $\dot{x}(t)$ from $x(t)$ and $u(t)$ for the $i$ th subinterval of $T_{s} . C_{i}^{\mathrm{T}}$ and $E_{i}$ are the output and feedthrough matrices, respectively, that extract a desired output $y(t)$ from $x(t)$ and $u(t)$ for the $i$ th subinterval of $T_{s}$. Solving the state Equation (7a) at the time boundaries of the $i$-th subinterval leads to the following difference equation:

$$
x\left(t_{i+1}\right)=\mathrm{e}^{A_{i}\left(t_{i+1}-t_{i}\right)} x\left(t_{i}\right)+\int_{t_{i}}^{t_{i+1}} \mathrm{e}^{A_{i}\left(t_{i+1}-\tau\right)} B_{i} u(\tau) \mathrm{d} \tau
$$

where $t_{i}=T_{i}+\hat{t}_{i} . T_{i}$ is the DC component of $t_{i}$ and $\hat{t}_{i}$ is the AC component which is nonzero when a perturbation is introduced to the system. For DC analysis no perturbations are considered making $t_{i}=T_{i}, x(t)=X(t)$, and $u(t)=U$. The $\mathrm{DC}$ form of $(7 \mathrm{ab})$ is:

$$
\dot{X}(t)=A_{i} X(t)+B_{i} U
$$




$$
Y(t)=C_{i}^{\mathrm{T}} X(t)+E_{i} U
$$

and the DC form of (8) is:

$$
X\left(T_{i+1}\right)=\mathrm{e}^{A_{i}\left(T_{i+1}-T_{i}\right)} X\left(T_{i}\right)+\int_{T_{i}}^{T_{i+1}} \mathrm{e}^{A_{i}\left(T_{i+1}-\tau\right)} B_{i} U \mathrm{~d} \tau
$$

Evaluating the integral results in:

$$
X\left(T_{i+1}\right)=\Phi_{i} X\left(T_{i}\right)+\Psi_{i} U
$$

where

$$
\begin{gathered}
\Phi_{i}=\mathrm{e}^{A_{i}\left(T_{i+1}-T_{i}\right)} \\
\Psi_{i}=A_{i}^{-1}\left[\Phi_{i}-I\right] B_{i}
\end{gathered}
$$

for $i \in\left(1,2, \cdots, N_{s}\right)$, which is the DC difference equation. Equation (11a) will be solved for a finite $N_{s}$ as it will be required for later use. The case covered in this paper, i.e. CCM, there are two topologies so (11a) will only be given here for $N_{s}=2$. Doing so makes use of the following condition for a converter in large signal steady state:

$$
X(t)=X\left(t+n T_{s}\right)
$$

for any nonnegative integer $n$. For $N_{s}=2$, (11a) becomes:

$$
\begin{aligned}
& X\left(T_{2}\right)=\Phi_{1} X\left(T_{1}\right)+\Psi_{1} U \\
& X\left(T_{3}\right)=\Phi_{2} X\left(T_{2}\right)+\Psi_{2} U
\end{aligned}
$$

From (12) we see that $X\left(T_{3}\right)=X\left(T_{1}\right)$ in DC steady state for $N_{s}=2$, Equation (13a) can be substituted into (13b) and $X\left(T_{1}\right)$ can be solved for:

$$
X\left(T_{1}\right)=\left(I-\Phi_{2} \Phi_{1}\right)^{-1}\left(\Phi_{2} \Psi_{1}+\Psi_{2}\right) U
$$

With $X\left(T_{1}\right)$ solved for, (14) can then be substituted into (13a) to obtain an expression for $X\left(T_{2}\right)$.

To obtain the small signal difference equation, the state vector $x\left(t_{i}\right)$ and switching time $t_{i}$ are first split into their DC and AC components:

$$
x\left(t_{i}\right)=X\left(t_{i}\right)+\hat{x}\left(t_{i}\right)=X\left(T_{i}+\hat{t}_{i}\right)+\hat{x}\left(T_{i}+\hat{t}_{i}\right)
$$

Taking the Taylor series expansions of the terms in (15):

$$
\begin{aligned}
& X\left(T_{i}+\hat{t}_{i}\right) \approx X\left(T_{i}\right)+\dot{X}\left(T_{i}\right) \hat{t}_{i} \\
& \hat{x}\left(T_{i}+\hat{t}_{i}\right) \approx \hat{x}\left(T_{i}\right)+\dot{\hat{x}}\left(T_{i}\right) \hat{t}_{i}
\end{aligned}
$$

Combining (15) and (16):

$$
x\left(t_{i}\right) \approx X\left(T_{i}\right)+\dot{X}\left(T_{i}\right) \hat{t}_{i}+\hat{x}\left(T_{i}\right)+\dot{\hat{x}}\left(T_{i}\right) \hat{t}_{i}
$$

Dropping all but the first order (small signal) terms and substituting (9a) gives $\hat{x}\left(t_{i}\right)$ and puts it in a form that will be convenient later on:

$$
\hat{x}\left(t_{i}\right) \approx \dot{X}\left(T_{i}\right) \hat{t}_{i}+\hat{x}\left(T_{i}\right)=\left[A_{i} X\left(T_{i}\right)+B_{i} U\right] \hat{t}_{i}+\hat{x}\left(T_{i}\right)
$$

The input is also perturbed to get:

$$
u(t)=U+\hat{u}(t)
$$


where $U$ is the constant DC component of $u(t)$ and $\hat{u}(t)$ is the AC component which will be chosen to take the form of $\hat{u}(t)=\hat{u}_{p} \mathrm{e}^{j \omega t}$, where $\hat{u}_{p}$ is the peak value of the perturbation. The reasons for choosing an exponential form for the excitation signal is that the real component of $\mathrm{e}^{j \omega t}$ is a cosine wave and the exponential form will be convenient later on in solving the small signal difference equation for $\hat{x}\left(T_{i}\right)$. For similar reasons, all perturbations in this paper will be chosen to be of the same form. Symbol $\omega$ represents the frequency of the perturbation signal in radians/second. Substituting (19) into (8) gives:

$$
x\left(t_{i+1}\right)=\mathrm{e}^{A_{i}\left(t_{i+1}-t_{i}\right)} x\left(t_{i}\right)+\int_{t_{i}}^{t_{i+1}} \mathrm{e}^{A_{i}\left(t_{i+1}-\tau\right)} B_{i} U \mathrm{~d} \tau+\int_{t_{i}}^{t_{i+1}} \mathrm{e}^{A_{i}\left(t_{i+1}-\tau\right)} B_{i} \hat{u}_{p} \mathrm{e}^{j \omega \tau} \mathrm{d} \tau
$$

Evaluating the integral yields:

$$
\begin{aligned}
x\left(t_{i+1}\right)= & \mathrm{e}^{A_{i}\left(t_{i+1}-t_{i}\right)} x\left(t_{i}\right)+A_{i}^{-1}\left[\mathrm{e}^{A_{i}\left(t_{i+1}-t_{i}\right)}-I\right] B_{i} U \\
& +\left(j \omega-A_{i}\right)^{-1} \mathrm{e}^{j \omega t_{i+1}}\left[I-\mathrm{e}^{-\left(j \omega I-A_{i}\right)\left(t_{i+1}-t_{i}\right)}\right] B_{i} \hat{u}_{p}
\end{aligned}
$$

Substituting $t_{i}=T_{i}+\hat{t}_{i}$ and $t_{i+1}=T_{i+1}+\hat{t}_{i+1}$ into (21) and denoting $\chi_{i}=j \omega I-A_{i}$ and $\beta_{i}=\mathrm{e}^{-j \omega\left(T_{i+1}-T_{i}\right)} \Phi_{i}$ results in:

$$
\begin{aligned}
x\left(t_{i+1}\right)= & \Phi_{i} \mathrm{e}^{A_{i}\left(\hat{t}_{i+1}-\hat{t}_{i}\right)} x\left(t_{i}\right)+A_{i}^{-1}\left[\Phi_{i} \mathrm{e}^{A_{i}\left(\hat{t}_{i+1}-\hat{t}_{i}\right)}-I\right] B_{i} U \\
& +\chi_{i}^{-1} \mathrm{e}^{j \omega T_{i+1}} \mathrm{e}^{j \omega \hat{t}_{i+1}}\left[I-\beta_{i} \mathrm{e}^{-\chi_{i}\left(\hat{t}_{i+1}-\hat{t}_{i}\right)}\right] B_{i} \hat{u}_{p}
\end{aligned}
$$

With time perturbations $\hat{t}_{i}$ and $\hat{t}_{i+1}$ appearing as exponents, the exponentials containing them will be linearized by replacing them with the first two terms of the Taylor series expansion:

$$
\mathrm{e}^{x} \approx 1+x
$$

which allows (22) to take the form:

$$
\begin{aligned}
x\left(t_{i+1}\right)= & \Phi_{i}\left[I+A_{i}\left(\hat{t}_{i+1}-\hat{t}_{i}\right)\right] x\left(t_{i}\right)+A_{i}^{-1}\left\{\Phi_{i}\left[I+A_{i}\left(\hat{t}_{i+1}-\hat{t}_{i}\right)\right]-I\right\} B_{i} U \\
& +\chi_{i}^{-1} \mathrm{e}^{j \omega T_{i+1}}\left(1+j \omega \hat{t}_{i+1}\right)\left\{I-\beta_{i}\left[I+\chi_{i}\left(\hat{t}_{i+1}-\hat{t}_{i}\right)\right]\right\} B_{i} \hat{u}_{p}
\end{aligned}
$$

Application of (23) to (22) is the first step in linearizing this equation. This results in (24) which is an approximation however DC and AC small signal models only consider zeroth and first order terms, respectively, thus no accuracy is lost in the "small signal sense". Substituting in (17) for $x\left(t_{i}\right)$ and $x\left(t_{i+1}\right)$, dropping any terms that are not small signal terms (first order), and solving for $\hat{x}\left(T_{i+1}\right)$ :

$$
\begin{aligned}
\hat{x}\left(T_{i+1}\right)= & \Phi_{i} \hat{x}\left(T_{i}\right)+\left[\left(A_{i}-A_{i+1}\right) X\left(T_{i+1}\right)+\left(B_{i}-B_{i+1}\right) U\right] \hat{t}_{i+1} \\
& +\chi_{i}^{-1}\left[I-\beta_{i}\right] B_{i} \hat{u}_{p} \mathrm{e}^{j \omega T_{i+1}}
\end{aligned}
$$

The small signal difference equation will later be solved for a finite $N_{s}$.

In summary, the difference equations developed in this section along with some important identities are, for $i \in\left(1,2, \cdots, N_{s}\right)$ :

DC Steady State Difference Equation (from Equation (11a)):

$$
X\left(T_{i+1}\right)=\Phi_{i} X\left(T_{i}\right)+\Psi_{i} U
$$

AC Small Signal Difference Equation (from Equation (25)): 


$$
\hat{x}\left(T_{i+1}\right)=\Phi_{i} \hat{x}\left(T_{i}\right)+\xi_{i} \hat{t}_{i+1}+\eta_{i} B_{i} \hat{u}\left(T_{i+1}\right)
$$

where

$$
\begin{aligned}
& \Phi_{i}=\mathrm{e}^{A_{i}\left(T_{i+1}-T_{i}\right)} \\
& \Psi_{i}=A_{i}^{-1}\left[\Phi_{i}-I\right] B_{i} \\
& \chi_{i}=j \omega I-A_{i} \\
& \beta_{i}=\mathrm{e}^{-j \omega\left(T_{i+1}-T_{i}\right)} \Phi_{i} \\
& \eta_{i}=\chi_{i}^{-1}\left(I-\beta_{i}\right) \\
& \xi_{i}=\left(A_{i}-A_{i+1}\right) X\left(T_{i+1}\right)+\left(B_{i}-B_{i+1}\right) U
\end{aligned}
$$

The value of $X\left(T_{1}\right)$, for $N_{s}=2$, is given by (14), which is reproduced below:

$$
X\left(T_{1}\right)=\left(I-\Phi_{2} \Phi_{1}\right)^{-1}\left(\Phi_{2} \Psi_{1}+\Psi_{2}\right) U
$$

Another useful identity is:

$$
\gamma_{i}=\dot{X}\left(T_{i+1}\right)+\xi_{i}=A_{i} X\left(T_{i+1}\right)+B_{i} U
$$

The following scalars or vectors may be split into their DC and AC components:

$$
\begin{aligned}
& t_{i}=T_{i}+\hat{t}_{i} \\
& d=D+\hat{d}(t) \\
& x(t)=X(t)+\hat{x}(t) \\
& u(t)=U+\hat{u}(t) \\
& r(t)=R+\hat{r}(t)
\end{aligned}
$$

where all perturbation signals take the following form:

$$
\begin{aligned}
& \hat{d}(t)=\hat{d}_{p} \mathrm{e}^{j \omega t} \\
& \hat{u}(t)=\hat{u}_{p} \mathrm{e}^{j \omega t} \\
& \hat{r}(t)=\hat{r}_{p} \mathrm{e}^{j \omega t}
\end{aligned}
$$

where $\hat{d}_{p}, \hat{u}_{p}$, and $\hat{r}_{p}$ are the peak magnitudes of the perturbations. It should be noted that because $u(t)$ is a scalar containing only $v_{g}(t), \hat{u}_{p}$ is the peak magnitude of $\hat{v}_{g}(t)$. Also, the following holds for a small signal state vector in large signal steady state in the presence of exponential perturbations:

$$
\hat{x}(t) \mathrm{e}^{j \omega n T_{s}}=\hat{x}\left(t+n T_{s}\right)
$$

for all positive $n$, which relates $\hat{x}(t)$ to $\hat{x}\left(t+n T_{s}\right)$ for all $t$.

\section{The Exact Small Signal Transfer Function Expression}

A general expression for the transfer functions of interest will be derived in this section by taking the average of the time varying function of a periodic network. This general expression will represent the frequency response of time interval modulated switched networks. The time varying transfer function is defined as:

$$
H(j \omega, t)=\int_{-\infty}^{\infty} h(t, \tau) \mathrm{e}^{-j \omega \tau} \mathrm{d} \tau
$$

where $h(t, \tau)$ is the impulse response at time $t$ caused by an impulse at time 
$t-\tau$. The output of the system $y(t)$ can be found by generalizing (33) for any input $u(t)$ :

$$
y(t)=\int_{-\infty}^{\infty} h(t, \tau) u(t-\tau) \mathrm{d} \tau
$$

Considering an input of the form $u(t)=\mathrm{e}^{j \omega t}$ as in (31) results in:

$$
\begin{aligned}
y(t) & =\int_{-\infty}^{\infty} h(t, \tau) \mathrm{e}^{j \omega(t-\tau)} \mathrm{d} \tau \\
& =\left[\int_{-\infty}^{\infty} h(t, \tau) \mathrm{e}^{-j \omega \tau} \mathrm{d} \tau\right] \mathrm{e}^{j \omega t} \\
& =H(j \omega, t) \mathrm{e}^{j \omega t}
\end{aligned}
$$

Rearranging gives a useful definition that will be used later:

$$
H(j \omega, t)=\left.\frac{y(t)}{u(t)}\right|_{u(t)=\mathrm{e}^{j \omega t}}
$$

The relation between $u(t)$ and its Fourier transform $U(j \omega)$ is:

$$
u(t)=\frac{1}{2 \pi} \int_{-\infty}^{\infty} U(j \omega) \mathrm{e}^{j \omega t} \mathrm{~d} \omega
$$

where

$$
U(j \omega)=2 \pi \delta(\omega)
$$

and $\delta(\omega)$ is the Dirac delta function. Substituting (37) into (34) yields:

$$
\begin{aligned}
y(t) & =\int_{-\infty}^{\infty} h(t, \tau)\left[\frac{1}{2 \pi} \int_{-\infty}^{\infty} U(j \omega) \mathrm{e}^{j \omega(t-\tau)} \mathrm{d} \omega\right] \mathrm{d} \tau \\
& =\frac{1}{2 \pi} \int_{-\infty}^{\infty}\left[\int_{-\infty}^{\infty} h(t, \tau) \mathrm{e}^{-j \omega \tau} \mathrm{d} \tau\right] U(j \omega) \mathrm{e}^{j \omega t} \mathrm{~d} \omega \\
& =\frac{1}{2 \pi} \int_{-\infty}^{\infty} H(j \omega, t) U(j \omega) \mathrm{e}^{j \omega t} \mathrm{~d} \omega
\end{aligned}
$$

From (39) the output frequency spectrum $Y(j \omega)$ can be found. In taking the Fourier transform of (39), a dummy variable $\mu$ will be used to avoid confusion:

$$
\begin{aligned}
Y(j \mu) & =\int_{-\infty}^{\infty} y(t) \mathrm{e}^{-j \mu t} \mathrm{~d} t \\
& =\int_{-\infty}^{\infty}\left[\frac{1}{2 \pi} \int_{-\infty}^{\infty} H(j \omega, t) U(j \omega) \mathrm{e}^{j \omega t} \mathrm{~d} \omega\right] \mathrm{e}^{-j \mu t} \mathrm{~d} t \\
& =\frac{1}{2 \pi} \int_{-\infty}^{\infty}\left[\int_{-\infty}^{\infty} H(j \omega, t) \mathrm{e}^{j \omega t} \mathrm{e}^{-j \mu t} \mathrm{~d} t\right] U(j \omega) \mathrm{d} \omega \\
& =\int_{-\infty}^{\infty} \int_{-\infty}^{\infty} H(j \omega, t) \mathrm{e}^{j \omega t} \delta(\omega) \mathrm{d} \omega \mathrm{e}^{-j \mu t} \mathrm{~d} t
\end{aligned}
$$

After applying the sifting property to (40):

$$
Y(j \mu)=\int_{-\infty}^{\infty} H(0, t) \mathrm{e}^{-j \mu t} \mathrm{~d} t
$$

Partitioning the time axis into an infinite number of switching periods indexed by $k$ and then further subdividing the switching periods into $N_{s}$ subintervals of length $t_{i+1}-t_{i}$ results in:

$$
Y(j \mu)=\sum_{k=-\infty}^{\infty} \sum_{i=1}^{N_{s}} \int_{t_{i}}^{t_{i+1}} H(0, t) \mathrm{e}^{-j \mu t} \mathrm{~d} t
$$


Noting from (7ab) and (36) that:

$$
H(0, t)=y(t)=C_{i}^{\mathrm{T}} x(t)+E_{i} u(t)
$$

Equation (42) becomes:

$$
Y(j \mu)=\sum_{k=-\infty}^{\infty} \sum_{i=1}^{N_{s}} \int_{t_{i}}^{t_{i+1}}\left[C_{i}^{\mathrm{T}} x(t)+E_{i} u(t)\right] \mathrm{e}^{-j \mu t} \mathrm{~d} t
$$

Substituting in (17) and (19) into (44), evaluating the integral, linearizing the terms with $\hat{t}_{i}$ and $\hat{t}_{i+1}$, and dropping all of the terms that are not small signal terms (first order) results in:

$$
\hat{Y}(j \omega)=\sum_{k=-\infty}^{\infty} \sum_{i=1}^{N_{s}} \mathrm{e}^{-j \omega T_{i}}\left[C_{i}^{\mathrm{T}} \eta_{i} \hat{x}\left(T_{i}\right)+\kappa_{i} \hat{t}_{i}+\lambda_{i} \hat{t}_{i+1}+\rho_{i} \hat{u}\left(T_{i}\right)\right]
$$

where

$$
\begin{aligned}
& \eta_{i}=\chi_{i}^{-1}\left(I-\beta_{i}\right) \\
& \kappa_{i}=-\left[C_{i}^{\mathrm{T}} X\left(T_{i}\right)+E_{i} U\right] \\
& \lambda_{i}=\left[C_{i}^{\mathrm{T} X}\left(T_{i+1}\right)+E_{i} U\right] \mathrm{e}^{j \omega\left(T_{i+1}-T_{i}\right)} \\
& \rho_{i}=C_{i}^{\mathrm{T}} \chi_{i}^{-1}\left[\left(T_{i+1}-T_{i}\right) I-\eta_{i}\right] B_{i}+E_{i}\left(T_{i+1}-T_{i}\right)
\end{aligned}
$$

The expression (45) can be simplified by examining the terms of the summation regarding $\hat{t}_{i}$ and $\hat{t}_{i+1}$ :

$$
\sum_{i=1}^{N_{s}} \mathrm{e}^{-j \omega T_{i}}\left(\kappa_{i} \hat{t}_{i}+\lambda_{i} \hat{t}_{i+1}\right)
$$

The summation (47) can be expanded into:

$$
\begin{aligned}
& \sum_{i=1}^{N_{s}} \mathrm{e}^{-j \omega T_{i}}\left(\kappa_{i} \hat{t}_{i}+\lambda_{i} \hat{t}_{i+1}\right) \\
& =\mathrm{e}^{-j \omega T_{i}} \kappa_{i} \hat{t}_{i}+\mathrm{e}^{-j \omega T_{N_{s}}} \lambda_{N_{s}} \hat{t}_{N_{s}}+\sum_{i=1}^{N_{s}-1}\left(\mathrm{e}^{-j \omega T_{i}} \lambda_{i}+\mathrm{e}^{-j \omega T_{i+1}} \kappa_{i+1}\right) \hat{t}_{i+1}
\end{aligned}
$$

Further expanding the first two terms of (48):

$$
\begin{aligned}
& \mathrm{e}^{-j \omega T_{i}} \kappa_{i} \hat{t}_{i}+\mathrm{e}^{-j \omega T_{N_{s}}} \lambda_{N_{s}} \hat{t}_{N_{s}} \\
& =\mathrm{e}^{-j \omega T_{i}}\left\{-\left[C_{1}^{\mathrm{T}} X\left(T_{i}\right)+E_{1} U\right]\right\} \hat{t_{1}} \\
& +\mathrm{e}^{-j \omega T_{N_{s}}}\left[C_{N_{s}}^{\mathrm{T}} X\left(T_{N_{s}+1}\right)+E_{N_{s}} U\right] \mathrm{e}^{-j \omega\left(T_{N_{s}+1}-T_{N_{s}}\right)} \hat{t}_{N_{s}+1}
\end{aligned}
$$

Because the system is periodic and cycles through $N_{s}$ topologies, the following relations hold:

$$
\begin{aligned}
& C_{1}^{\mathrm{T}}=C_{N_{s}+1}^{\mathrm{T}} \\
& E_{1}=E_{N_{s}+1} \\
& \hat{t}_{1}=\hat{t}_{N_{s}+1} \mathrm{e}^{-j \omega\left(T_{N_{s}+1}-T_{1}\right)} \\
& X\left(T_{1}\right)=X\left(T_{N_{s}+1}\right)
\end{aligned}
$$

Letting $\zeta_{i}=\left(C_{i}^{\mathrm{T}}-C_{i+1}^{\mathrm{T}}\right) X\left(T_{i+1}\right)+\left(E_{i}-E_{i+1}\right) U$, Equation (49) becomes: 


$$
\mathrm{e}^{-j \omega T_{i}} \kappa_{i} \hat{t}_{i}+\mathrm{e}^{-j \omega T_{N_{s}}} \lambda_{N_{s}} \hat{t}_{N_{s}}=\mathrm{e}^{-j \omega T_{N_{s}+1}} \zeta_{N_{s}} \hat{t}_{N_{s}+1}
$$

and the last term of (48) becomes:

$$
\sum_{i=1}^{N_{s}-1}\left(\mathrm{e}^{-j \omega T_{i}} \lambda_{i}+\mathrm{e}^{-j \omega T_{i+1}} \kappa_{i+1}\right) \hat{t}_{i+1}=\sum_{i=1}^{N_{s}-1} \mathrm{e}^{-j \omega T_{i+1}} \zeta_{i} \hat{t}_{i+1}
$$

Combining (51) and (52) allows (47) to be expressed as:

$$
\sum_{i=1}^{N_{s}} \mathrm{e}^{-j \omega T_{i}}\left(\kappa_{i} \hat{t}_{i}+\lambda_{i} \hat{t}_{i+1}\right)=\sum_{i=1}^{N_{s}} \mathrm{e}^{-j \omega T_{i+1}} \zeta_{i} \hat{t}_{i+1}
$$

and (45) to take the final simplified form:

$$
\hat{Y}(j \omega)=\sum_{k=-\infty}^{\infty} \sum_{i=1}^{N_{s}} \mathrm{e}^{-j \omega T_{i}}\left[C_{i}^{\mathrm{T}} \eta_{i} \hat{x}\left(T_{i}\right)+\mathrm{e}^{-j \omega\left(T_{i+1}-T_{i}\right)} \zeta_{i} \hat{t}_{i+1}+\rho_{i} \hat{u}\left(T_{i}\right)\right]
$$

Note that Equation (54) is a simplified version of one previously derived in [6] (Equation (9)), where now only the time perturbation at the end of each subinterval is required. This equation is further simplified next leading to the general expression of the frequency response being sought.

While $\hat{Y}(j \omega)$ is the entire output spectrum containing impulses at integer multiples of $\omega$, we are only interested in the results at $\omega$ which is the frequency response $H_{0}(j \omega) . H_{0}(j \omega)$ can be obtained by taking the average of the inner summation over a period $T_{X}$ rather than the entire output spectrum. Figure 3 shows that $T_{x}$ can be an integer $N$ multiple of $T_{s}$, therefore:

$$
H_{0}(j \omega)=\frac{1}{N T_{s}} \sum_{k=0}^{N-1} \sum_{i=1}^{N_{s}} \mathrm{e}^{-j \omega T_{i}}\left[C_{i}^{\mathrm{T}} \eta_{i} \hat{x}\left(T_{i}\right)+\mathrm{e}^{-j \omega\left(T_{i+1}-T_{i}\right)} \zeta_{i} \hat{t}_{i+1}+\rho_{i} \hat{u}\left(T_{i}\right)\right]
$$

While it appears from (55) that the outer summation will have to be evaluated $N$ times since there are small signal terms that differ between switching cycles, after substitution and evaluation of the inner summation it is evident that these terms will become independent of which $T_{s}$ they are evaluated for. Therefore, the outer summation will result in $N$ equivalent terms which are then divided by $N$. This is equivalent to setting $N=1$.

It will become evident that finding the transfer functions of interest involves first determining expressions for $\hat{t}_{i}$ for $i \in\left(1,2, \cdots, N_{s}\right)$, solving the difference equation to obtain an expression for $\hat{x}\left(T_{1}\right)$, and then substituting the results

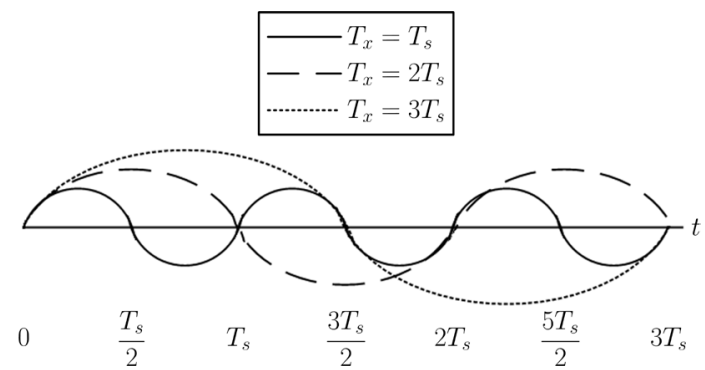

Figure 3. Period $T_{x}$ in the presence of perturbations, where the waveform of period $T_{s}$ can represent any output of the system while in steady state. While the shortest possible $T_{X}$ is not necessarily an integer $N$ multiple of $T_{s}$, the relation $T_{x}=N T_{s}$ does provide convenience. 
into the general expression $H_{0}(j \omega)$. After extracting $\frac{\hat{v}_{\text {out }}}{\hat{r}}$ and $\frac{\hat{v}_{\text {out }}}{\hat{v}_{g}}$ from $H_{0}(j \omega)$, the Bode plots of the transfer functions will be compared to those from a commercial simulator.

\section{Voltage Mode Transfer Functions}

The transfer functions of interest will now be derived for voltage mode (VM) DC-to-DC converters in CCM. As an example, the boost converter shown in Figure 4 will be used. The output voltage controller has been omitted in Figure 4 since the open loop transfer functions are derived, so the control signal $r(t)$ is externally supplied. The control signal $r(t)$ is compared to a sawtooth signal in order to generate a rectangular wave to directly control $Q$ and regulate the switching between topologies. A VM converter has its sawtooth signal externally supplied rather than, for example, being generated from some output of the system as for current mode control. So far, the start of the switching period $T_{s}$ has been defined by the period of the sawtooth signal. This way of defining the beginning of the $T_{s}$ will be called Model 1. Defining the start of the interval to be the switching event controlled by $r(t)$ instead, which will be called Model 2, will be considerably more convenient. For this reason, the derivations will take place in Model 2, and then the subscripts of the results rotated appropriately so that they apply to Model 1. Figure 5 shows very generally how Model 1 and Model 2 are related.

In the following the transfer functions $\frac{\hat{v}_{\text {out }}}{\hat{r}}$ and $\frac{\hat{v}_{\text {out }}}{\hat{u}}$ for the CCM operating mode will be derived and subsequently applied to a boost converter example.

A power converter operating in CCM implies that there are two topologies $\left(N_{s}=2\right)$ and thus two subintervals within a switching period $T_{s}$ Considering a CCM converter with Model 1, the first subinterval has an average (DC) length $D T_{s}$ and the second interval has a DC length $(1-D) T_{s}$. The effective topologies of the boost power stage during the two subintervals are shown in Figure 6. The switching period is described by Figure 7 in both Model 1 and Model 2. Model 2

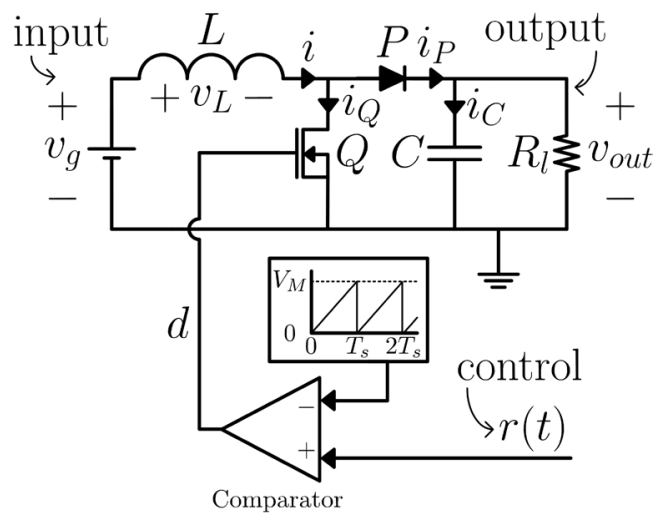

Figure 4. A VM boost converter. The control signal $r(t)$, externally supplied in this figure, is compared to a sawtooth signal of amplitude $V_{M}$ and switching period $T_{s}$ to create a rectangular wave with duty ratio $d$ that controls the switching between topologies. 


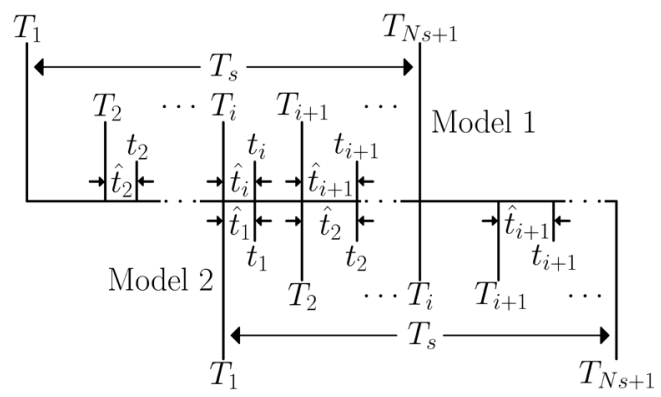

Figure 5. A general switching period $T_{s}$ described under Model 1 and Model 2. Under Model 1 the start and end of $T_{s}$ is defined by the switching period of the sawtooth signal, thus $\hat{t}_{1}=\hat{t}_{N_{s}+1}=0$, and the switching event $t_{i}$ is controlled by $r(t)$. Under Model 2, the start and end of $T_{s}$ is the switching event determined by $r(t)$.

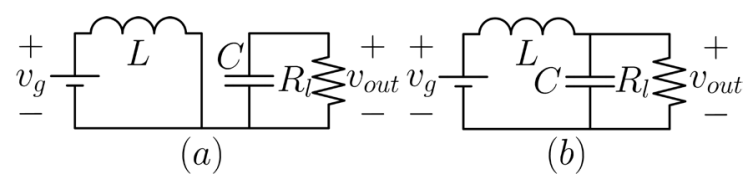

Figure 6. The effective topologies of a VM boost converter in CCM assuming ideal switches. (a) is the effective topology during the first subinterval of DC length $D T_{s}$ where $Q$ is on and $P$ is off. (b) is the effective topology during the second subinterval of DC length $(1-D) T_{s}$ where $Q$ is off and $P$ is on.

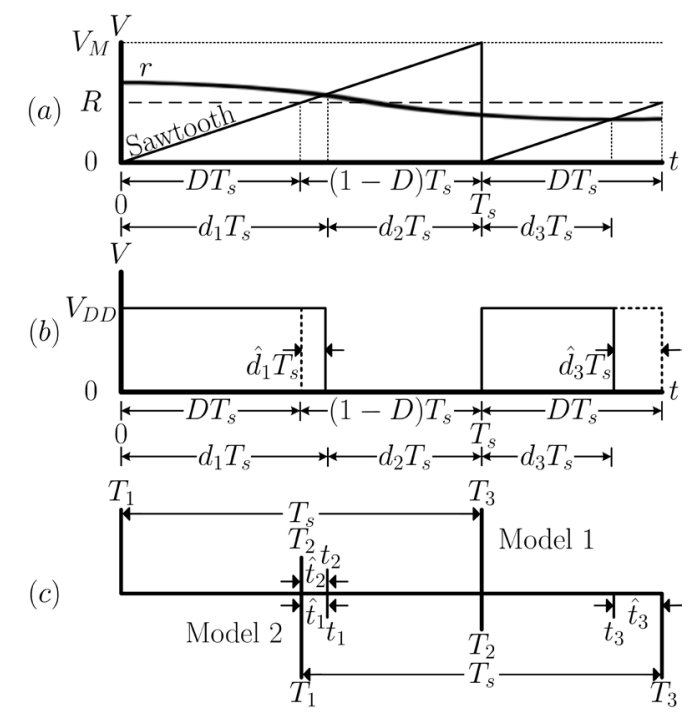

Figure 7. Switching period $T_{s}$ for a VM converter in CCM, described for both Model 1 and Model 2. (a) shows the sawtooth voltage of period $T_{s}$ being compared to the control signal $r(t)$ to produce the rectangular wave (b). (c) illustrates how the times are denoted under Model 1 and Model 2.

will be used for the following derivation, effectively swapping the order of the two subintervals. Considering Model 2, the switching time $t_{2}$ is determined by the period $T_{s}$ of the sawtooth signal and thus $\hat{t}_{2}=0 . t_{3}$ is the time when the sawtooth signal reaches the value of $r(t)$ : 


$$
t_{3}=\frac{T_{s} r\left(t_{3}\right)}{V_{M}}
$$

Dropping the DC components of $r\left(t_{3}\right)$ and $t_{3}$ :

$$
\hat{t}_{3}=\frac{T_{s} \hat{r}\left(t_{3}\right)}{V_{M}}
$$

Taking the Taylor series expansion of $\hat{r}\left(t_{3}\right)$ and dropping the second order term:

$$
\hat{r}\left(t_{3}\right)=\hat{r}\left(T_{3}+\hat{t}_{3}\right) \approx \hat{r}\left(T_{3}\right)+\dot{\hat{r}}\left(T_{3}\right) \hat{t}_{3} \approx \hat{r}\left(T_{3}\right)
$$

Substituting (58) into (57) gives an expression for $\hat{t}_{3}$ :

$$
\hat{t}_{3}=\frac{T_{s} \hat{r}\left(T_{3}\right)}{V_{M}}=\mu \hat{r}\left(T_{3}\right)
$$

where

$$
\mu=\frac{T_{s}}{V_{M}}
$$

With $\hat{t}_{3}$ obtained, the next step is to get $\hat{x}\left(T_{1}\right)$ by solving the difference Equation (27). From (27) an expression for $\hat{x}\left(T_{2}\right)$ is found:

$$
\hat{x}\left(T_{2}\right)=\Phi_{1} \hat{x}(T)_{1}+\eta_{1} B_{1} \hat{u}\left(T_{2}\right)
$$

Using (27) again and substituting in (59) and (61) gives:

$$
\begin{aligned}
\hat{x}\left(T_{3}\right) & =\Phi_{2} \hat{x}\left(T_{2}\right)+\xi_{2} \hat{t}_{3}+\eta_{2} B_{2} \hat{u}\left(T_{3}\right) \\
& =\Phi_{2} \Phi_{1} \hat{x}\left(T_{1}\right)+\xi_{2} \mu \hat{r}\left(T_{3}\right)+\Phi_{2} \eta_{1} B_{1} \hat{u}\left(T_{2}\right)+\eta_{2} B_{2} \hat{u}\left(T_{3}\right)
\end{aligned}
$$

Expressing all of the signals in their exponential form as in (31) and substituting in (32) for $\hat{x}\left(T_{3}\right)$ :

$$
\hat{x}\left(T_{1}\right) \mathrm{e}^{j \omega T_{s}}=\Phi_{2} \Phi_{1} \hat{x}\left(T_{1}\right)+\xi_{2} \mu \hat{r}_{p} \mathrm{e}^{j \omega T_{3}}+\Phi_{2} \eta_{1} B_{1} \hat{u}_{p} \mathrm{e}^{j \omega T_{2}}+\eta_{2} B_{2} \hat{u}_{p} \mathrm{e}^{j \omega T_{3}}
$$

Solving for $\hat{x}\left(T_{1}\right)$ :

$$
\hat{x}\left(T_{1}\right)=\left(I-\beta_{2} \beta_{1}\right)^{-1}\left[\xi_{2} \mu \hat{r}\left(T_{1}\right)+\left(\beta_{2} \eta_{1} B_{1}+\eta_{2} B_{2}\right) \hat{u}\left(T_{1}\right)\right]
$$

Now all that is left is to substitute (59), (61), and (64) into (55). Expanding (55) for $N_{s}=2$ gives:

$$
\begin{aligned}
H_{0}(j \omega)= & \frac{1}{T_{s}}\left[C_{1}^{\mathrm{T}} \eta_{1} \hat{x}\left(T_{1}\right) \mathrm{e}^{-j \omega T_{1}}+\rho_{1} \hat{u}_{p}+C_{2}^{\mathrm{T}} \eta_{2} \hat{x}\left(T_{2}\right) \mathrm{e}^{-j \omega T_{2}}\right. \\
& \left.+\zeta_{2} \hat{t}_{3} \mathrm{e}^{-j \omega T_{3}}+\rho_{2} \hat{u}_{p}\right]
\end{aligned}
$$

Substituting in (59):

$$
H_{0}(j \omega)=\frac{1}{T_{s}}\left[C_{1}^{\mathrm{T}} \eta_{1} \hat{x}\left(T_{1}\right) \mathrm{e}^{-j \omega T_{1}}+\zeta_{2} \mu \hat{r}_{p}+C_{2}^{\mathrm{T}} \eta_{2} \hat{x}\left(T_{2}\right) \mathrm{e}^{-j \omega T_{2}}+\left(\rho_{1}+\rho_{2}\right) \hat{u}_{p}\right]
$$

Now substituting in (61):

$$
\begin{aligned}
H_{0}(j \omega)= & \frac{1}{T_{s}}\left[\left(C_{1}^{\mathrm{T}} \eta_{1}+C_{2}^{\mathrm{T}} \eta_{2} \beta_{1}\right) \hat{x}\left(T_{1}\right) \mathrm{e}^{-j \omega T_{1}}+\zeta_{2} \mu \hat{r}_{p}\right. \\
& \left.+\left(C_{2}^{\mathrm{T}} \eta_{2} \eta_{1} B_{1}+\rho_{1}+\rho_{2}\right) \hat{u}_{p}\right]
\end{aligned}
$$


Finally substituting in (64):

$$
\begin{aligned}
H_{0}(j \omega)= & \frac{1}{T_{s}}\left\{\left[\left(C_{1}^{\mathrm{T}} \eta_{1}+C_{2}^{\mathrm{T}} \eta_{2} \beta_{1}\right)\left(I-\beta_{2} \beta_{1}\right)^{-1} \xi_{2} \mu+\zeta_{2} \mu\right] \hat{r}_{p}\right. \\
& +\left[\left(C_{1}^{\mathrm{T}} \eta_{1}+C_{2}^{\mathrm{T}} \eta_{2} \beta_{1}\right)\left(I-\beta_{2} \beta_{1}\right)^{-1}\left(\beta_{2} \eta_{1} B_{1}+\eta_{2} B_{2}\right)\right. \\
& \left.\left.+C_{2}^{\mathrm{T}} \eta_{2} \eta_{1} B_{1}+\rho_{1}+\rho_{2}\right] \hat{u}_{p}\right\}
\end{aligned}
$$

Rotating the subscripts of (68) to Model 1 only involves switching them, giving the final result:

$$
\begin{aligned}
H_{0}(j \omega)= & \frac{1}{T_{s}}\left\{\left[\left(C_{1}^{\mathrm{T}} \eta_{1} \beta_{2}+C_{2}^{\mathrm{T}} \eta_{2}\right)\left(I-\beta_{1} \beta_{2}\right)^{-1} \xi_{1} \mu+\zeta_{1} \mu\right] \hat{r}_{p}\right. \\
& +\left[\left(C_{1}^{\mathrm{T}} \eta_{1} \beta_{2}+C_{2}^{\mathrm{T}} \eta_{2}\right)\left(I-\beta_{1} \beta_{2}\right)^{-1}\left(\eta_{1} B_{1}+\beta_{1} \eta_{2} B_{2}\right)\right. \\
& \left.\left.+C_{1}^{\mathrm{T}} \eta_{1} \eta_{2} B_{2}+\rho_{1}+\rho_{2}\right] \hat{u}_{p}\right\}
\end{aligned}
$$

where

$$
\mu=\frac{T_{s}}{V_{M}}
$$

From (69) the transfer functions are extracted by setting the input of interest to one while setting the other to zero. The exact small-signal control-to-output transfer function $\frac{v_{\text {out }}}{\hat{r}}$ is therefore found by setting $\hat{r}_{p}=1$ and setting $\hat{u}_{p}=0$ :

$$
\frac{\hat{v}_{\text {out }}}{\hat{r}}=\frac{1}{V_{M}}\left[\left(C_{1}^{\mathrm{T}} \eta_{1} \beta_{2}+C_{2}^{\mathrm{T}} \eta_{2}\right)\left(I-\beta_{1} \beta_{2}\right)^{-1} \xi_{1}+\zeta_{1}\right]
$$
The exact small-signal input-to-output transfer function $\frac{\hat{v}_{\text {out }}}{\hat{v}_{g}}$ is found by
setting $\hat{r}_{p}=0$ and setting $\hat{u}_{p}=1$ :

$$
\begin{aligned}
\frac{\hat{v}_{\text {out }}}{\hat{v}_{g}}= & \frac{1}{T_{s}}\left[\left(C_{1}^{\mathrm{T}} \eta_{1} \beta_{2}+C_{2}^{\mathrm{T}} \eta_{2}\right)\left(I-\beta_{1} \beta_{2}\right)^{-1}\left(\eta_{1} B_{1}+\beta_{1} \eta_{2} B_{2}\right)\right. \\
& \left.+C_{1}^{\mathrm{T}} \eta_{1} \eta_{2} B_{2}+\rho_{1}+\rho_{2}\right]
\end{aligned}
$$

To relate these to formerly obtained results in the literature we will consider the following. The above transfer functions contain exponential terms. It can be readily shown that expanding these in a Taylor series and retaining only the first order terms results in the state space averaged transfer function models [8]. The state space averaged small-signal control-to-output transfer function is given by:

$$
\frac{\hat{v}_{\text {out }}}{\hat{r}}=\frac{1}{V_{M}}\left[\left(D C_{1}^{\mathrm{T}}+D^{\prime} C_{2}^{\mathrm{T}}\right)\left(j \omega I-\left(D A_{1}+D^{\prime} A_{2}\right)\right)^{-1} \xi_{1}+\zeta_{1}\right]
$$

and, the state space averaged small-signal input-to-output transfer function is given by:

$$
\frac{\hat{v}_{\text {out }}}{\hat{v}_{g}}=\left(D C_{1}^{\mathrm{T}}+D^{\prime} C_{2}^{\mathrm{T}}\right)\left(j \omega I-\left(D A_{1}+D^{\prime} A_{2}\right)\right)^{-1}\left(D B_{1}+D^{\prime} B_{2}\right)+\left(D E_{1}+D^{\prime} E_{2}\right)
$$

Comparison of these reduced order models with the exact models is discussed below. 


\section{Example}

A boost converter with the following parameters is used to compare the frequency responses obtained using the PSIM simulator [9] and the derived models.

$$
\begin{aligned}
& T_{s}=10 \mu \mathrm{s}, \quad\left(f_{s}=100 \mathrm{kHz}\right) \\
& V_{g}=15 \mathrm{~V} \\
& D=0.25 \\
& L=58 \mu \mathrm{H} \\
& C=5.5 \mu \mathrm{F} \\
& R_{l}=18.6 \Omega
\end{aligned}
$$

The "AC Sweep" functionality in PSIM is used to obtain the frequency responses from this simulator. The PSIM circuit configuration used to obtain the control to output response is shown in Figure 8 . The $100 \mathrm{kHz}$ triangular waveform at the negative input of the comparator has a peak-to-peak amplitude of 1 V. At the positive input of the comparator a $0.25 \mathrm{~V} \mathrm{DC}$ voltage is added to the small amplitude perturbation source, Vsweep. This established a steady state duty ratio of 0.25 . The amplitude of the perturbation source needed to be adjusted to be small enough to not overdrive the converter, which yields inaccurate results, yet needs to be large enough to provide a measurable output signal at high frequency. A start and end amplitude value of $0.02 \mathrm{~V}$ was found to give good results. The frequency sweep was from $100 \mathrm{~Hz}$ to $45 \mathrm{kHz}$ (which is slightly less than half the switching frequency).

The magnitude and phase responses for the control-to-output function are shown in Figure 9 and Figure 10, respectively. The describing function model is given by Equation (71). In each figure, there are two plots drawn. The first plot shows the model response which is drawn in blue, subsequently the response obtained from PSIM is overlaid in red. The match is so close such that the red completely overwrites the previous plotted curve. The complete Matlab code used to run the models and produce the plots is shown as three functions in the

\section{Appendix.}

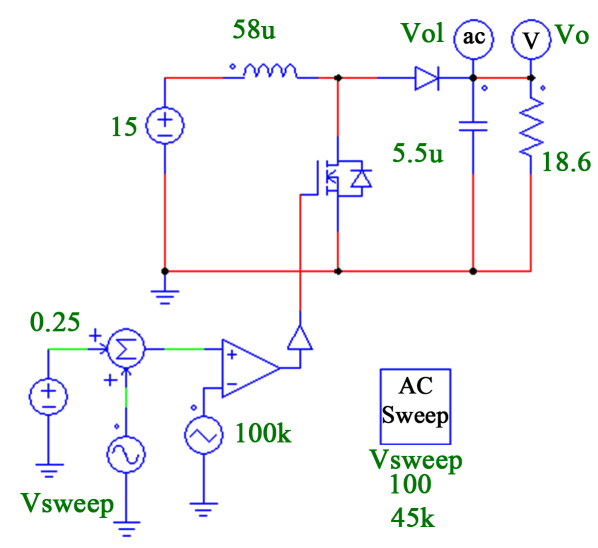

Figure 8. PSIM schematic used to obtain the control-to-output frequency response for the boost converter operating in CCM. 


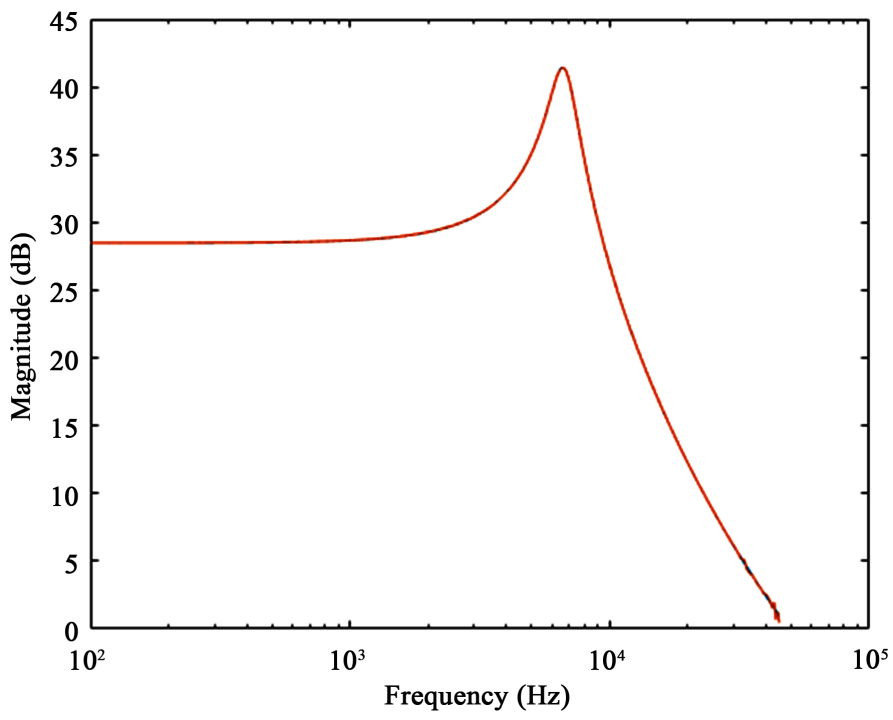

Figure 9. The magnitude response for the control to output transfer function of the boost converter operating in CCM. Model magnitude plot is in blue and the PSIM obtained magnitude plot is in red. We see excellent agreement such that only the red plot is mostly visible, since it is the second plot to be drawn.

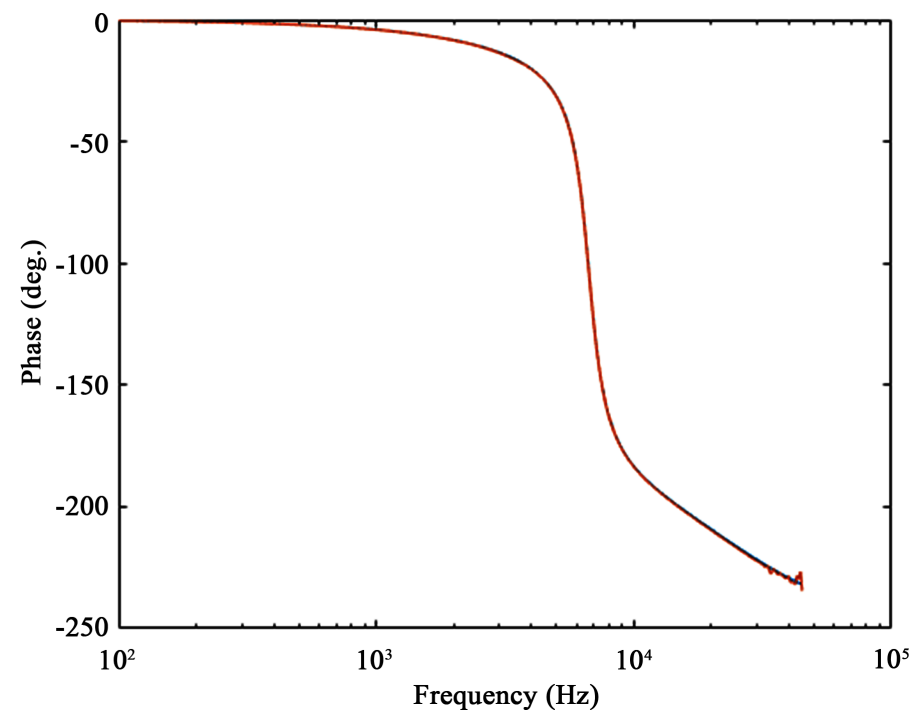

Figure 10. The phase response for the control to output transfer function of the boost converter operating in CCM. Model phase plot is in blue and the PSIM obtained phase plot is in red. We see excellent agreement such that only the red plot is mostly visible.

The PSIM schematic used to obtain the input voltage to output voltage response is shown in Figure 11. The perturbation source is now in series with the input voltage. A starting value of $0.05 \mathrm{~V}$ and end amplitude value of $0.3 \mathrm{~V}$ was used for the Vsweep perturbation signal. The magnitude and phase responses for the control-to-output function are shown in Figure 12 and Figure 13, respectively. The describing function model is given by Equation (78). Again the agreement between the derived model and the simulated result is seen to be excellent. These results validate the derived models. 


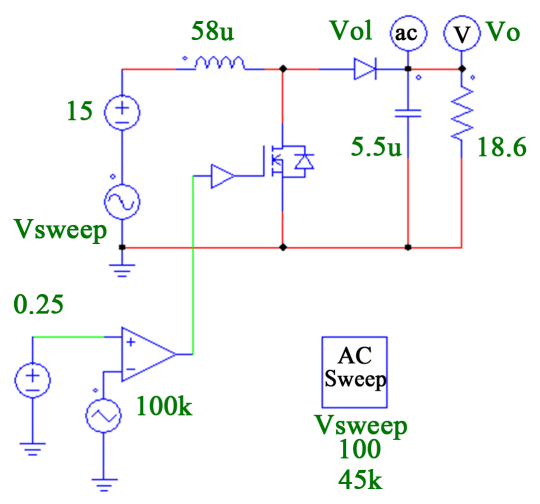

Figure 11. PSIM schematic used to obtain the input-to-output frequency response for the boost converter operating in CCM.

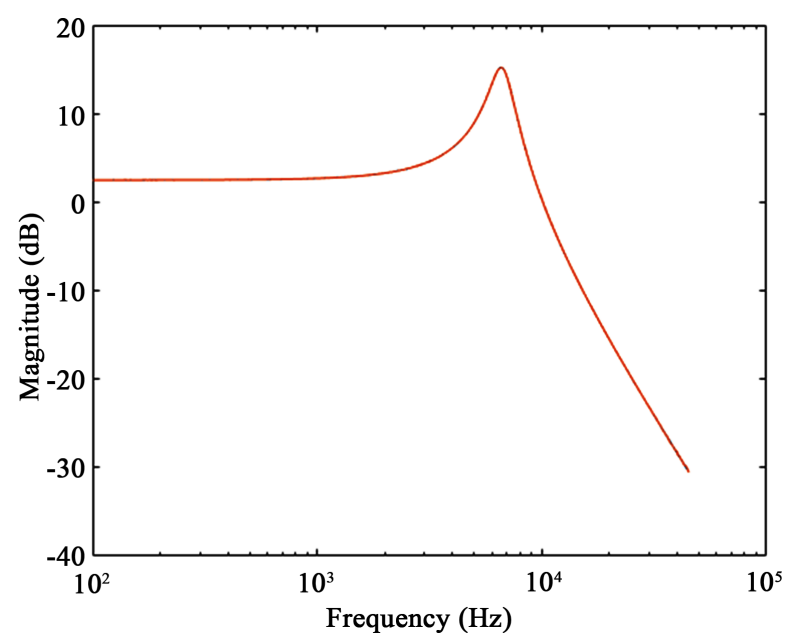

Figure 12. The magnitude response for the input to output transfer function of the boost converter operating in CCM. Model magnitude plot is in blue and the PSIM obtained magnitude plot is in red. We see excellent agreement such that only the red plot is mostly visible.

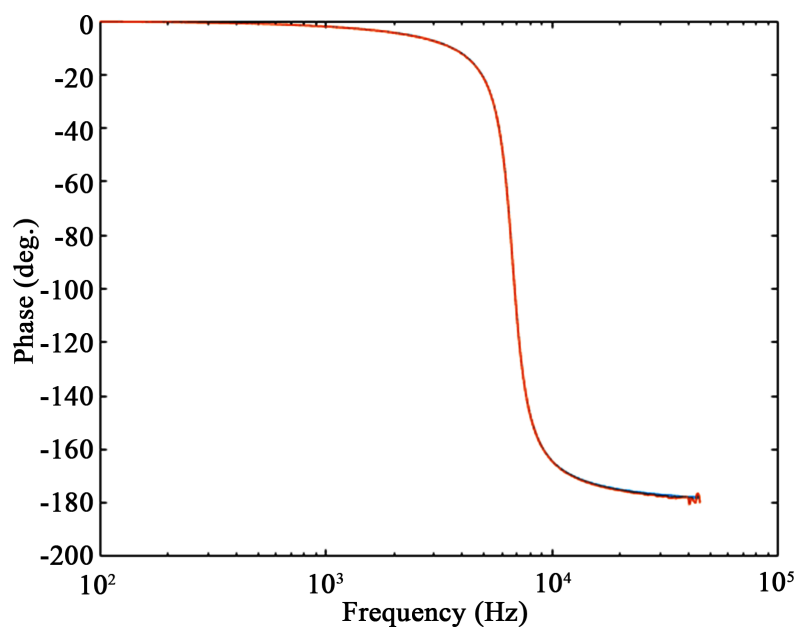

Figure 13. The phase response for the input to output transfer function of the boost converter operating in CCM. Model phase plot is in blue and the PSIM obtained phase plot is in red. We see excellent agreement such that only the red plot is mostly visible. 
Application of the reduced order (state space averaged) models, i.e. equations (73) and (74), for this example has shown that the accuracy obtained with these models is sufficient for this operating mode (i.e. CCM) and control regime (i.e. voltage mode control). Note however when one considers other operating modes, such as DCM, or other control schemes, such as current mode control, the low order averaged models lack sufficient accuracy at high frequencies. A wide range of models have been presented to model converters in DCM, for example, see [10] for a recent historical overview of these models.

\section{Conclusions}

Integral in control system design for dc-to-dc regulators is the use of transfer functions to determine relevant frequency responses. Typically average models have been used which may provide reasonable accuracy at low frequencies but often are inaccurate at high frequencies. In contrast a describing function model has been further developed in this paper. These models are based on a state equation formulation of the system. They are exact in the small-signal sense and therefore can precisely determine the frequency response over all excitation frequencies of interest. This modeling approach has been applied here to PWM converters operating in CCM in deriving control-to-output and input-to-output transfer functions.

An application example has been provided in the form of a boost dc-to-dc converter. The control to output voltage and input source voltage to output voltage frequency responses were determined. These were subsequently compared with the frequency responses obtained using the commercial simulator PSIM. An excellent match was achieved for the considered frequency range up to almost half the switching frequency. As the modeling method is exact, it may be used as a benchmark for accuracy by which other models may be compared and from which simplified models may be derived.

\section{Conflicts of Interest}

The authors declare no conflicts of interest regarding the publication of this paper.

\section{References}

[1] Chung, H.S.-H., Ionovici, A. and Zhang, J. (2000) Describing Functions of Power Electronics Circuits Using Progressive Analysis of Circuit Waveforms. IEEE Transactions on Circuits and Systems I: Fundamental Theory and Applications, 47, 1026-1037. https://doi.org/10.1109/81.855458

[2] Yue, X.L., Wang, X.F. and Blaabjerg, F. (2019) Review of Small-Signal Modeling Methods Including Frequency-Coupling Dynamics of Power Converters. IEEE Transactions on Power Electronics, 34, 3313-3328. https://doi.org/10.1109/TPEL.2018.2848980

[3] Yang, Z.C., Li, H., Liu, C., Ding, Y.H. and Zhang, B. (2019) Accurate Modeling and Stability Analysis for Chaotic PWM Boost Converters Based on Describing Func- 
tion Method. IECON 2019-45th Annual Conference of the IEEE Industrial Electronics Society, Lisbon, 14-17 October 2019, 1585-1590.

https://doi.org/10.1109/IECON.2019.8926913

[4] Tymerski, R. (1991) Frequency Analysis of Time-Interval-Modulated Switched Networks. IEEE Transactions on Power Electronics, 6, 287-295.

https://doi.org/10.1109/63.76815

[5] Tymerski, R. (1994) Application of the Time-Varying Transfer Function for Exact Small-Signal Analysis. IEEE Transactions on Power Electronics, 9, 196-205. https://doi.org/10.1109/63.286812

[6] Tymerski, R. (1997) Exact Input-to-Output Frequency Response of Time Interval Modulated Switched Networks. Power Electronics Specialists Conference, 1, 428-434.

[7] Zadeh, L.A. (1950) Frequency Analysis of Variable Networks. Proceedings of the IRE, 38, 291-299. https://doi.org/10.1109/JRPROC.1950.231083

[8] Middlebrook, R.D. and Cuk, S. (1976) A General Unified Approach to Modelling Switching-Converter Power Stages. 1976 IEEE Power Electronics Specialists Conference, Cleveland, 8-10 June 1976, 18-34.

https://doi.org/10.1109/PESC.1976.7072895

[9] https://powersimtech.com/

[10] Mao, Y.-J., Lam, C.-S., Sin, S.-W., Wong, M.-C., Martins, R.P. and Piegari, L. (2018) Review and Selection Strategy for High-Accuracy Modeling of PWM Converters in DCM. Journal of Electrical and Computer Engineering, 2018, Article ID: 3901693. https://doi.org/10.1155/2018/3901693 


\section{Appendix}

The appendix shows the Matlab code of the three functions used to determine and plot the frequency responses of the boost converter example (Figures A1-A3).

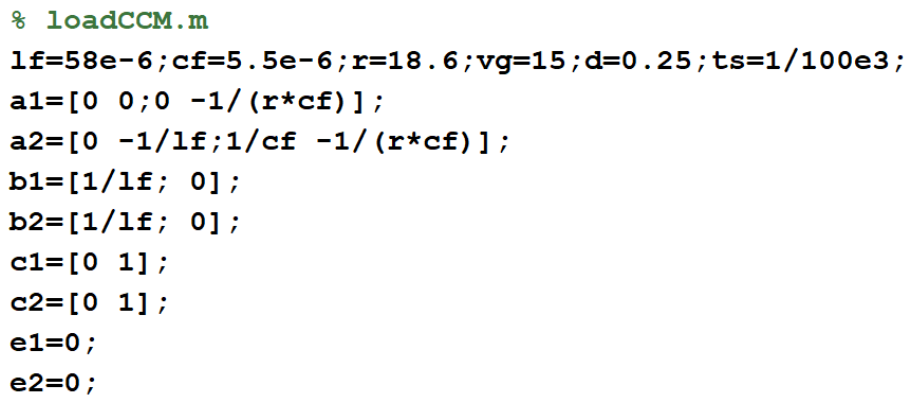

Figure A1. Matlab function: loadCCM.m. This function produces the state matrices for the boost converter.

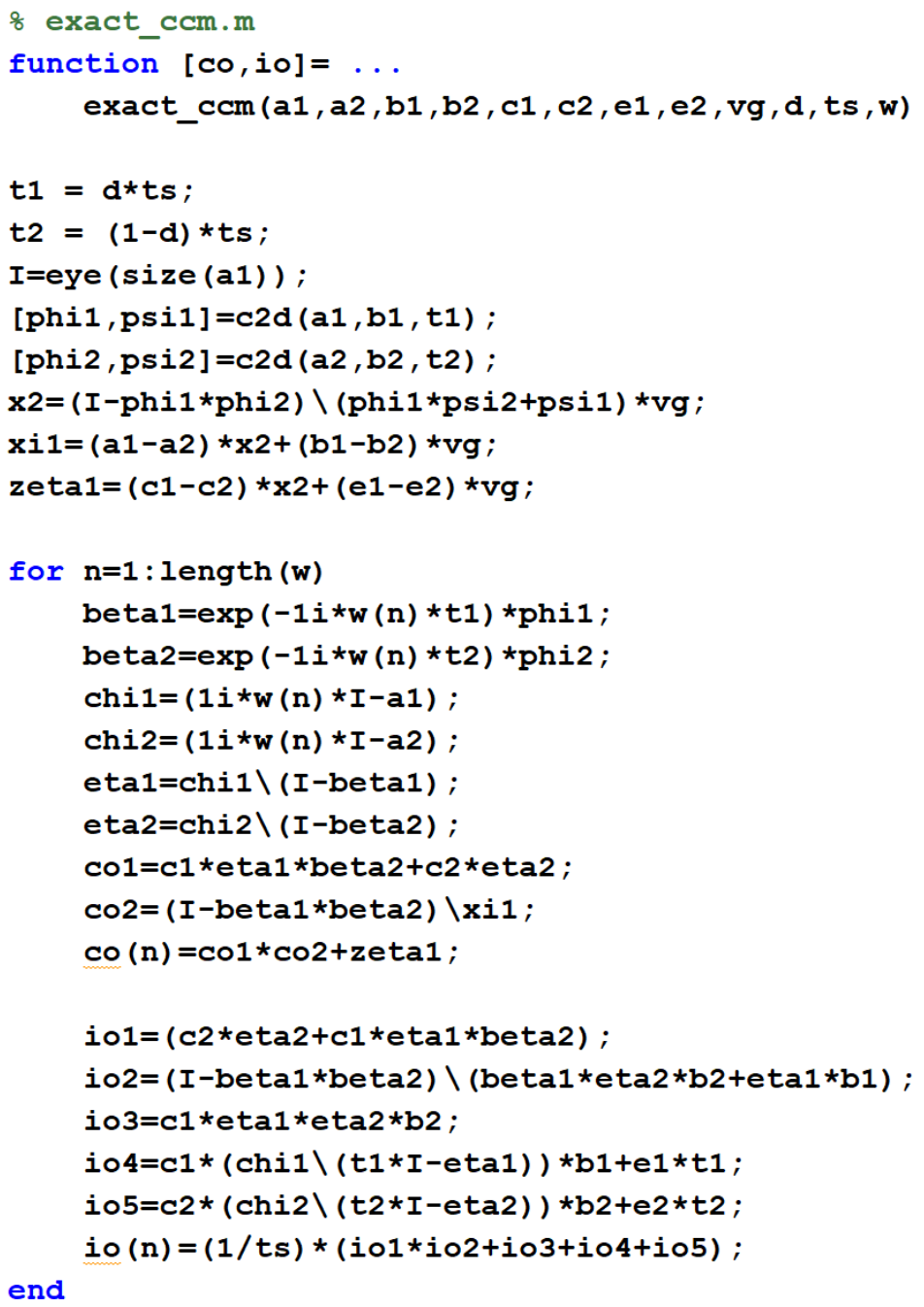

Figure A2. Matlab function: exact_ccm.m. Given the state matrices and other parameters, the control to output and input to output transfer functions are determined. 


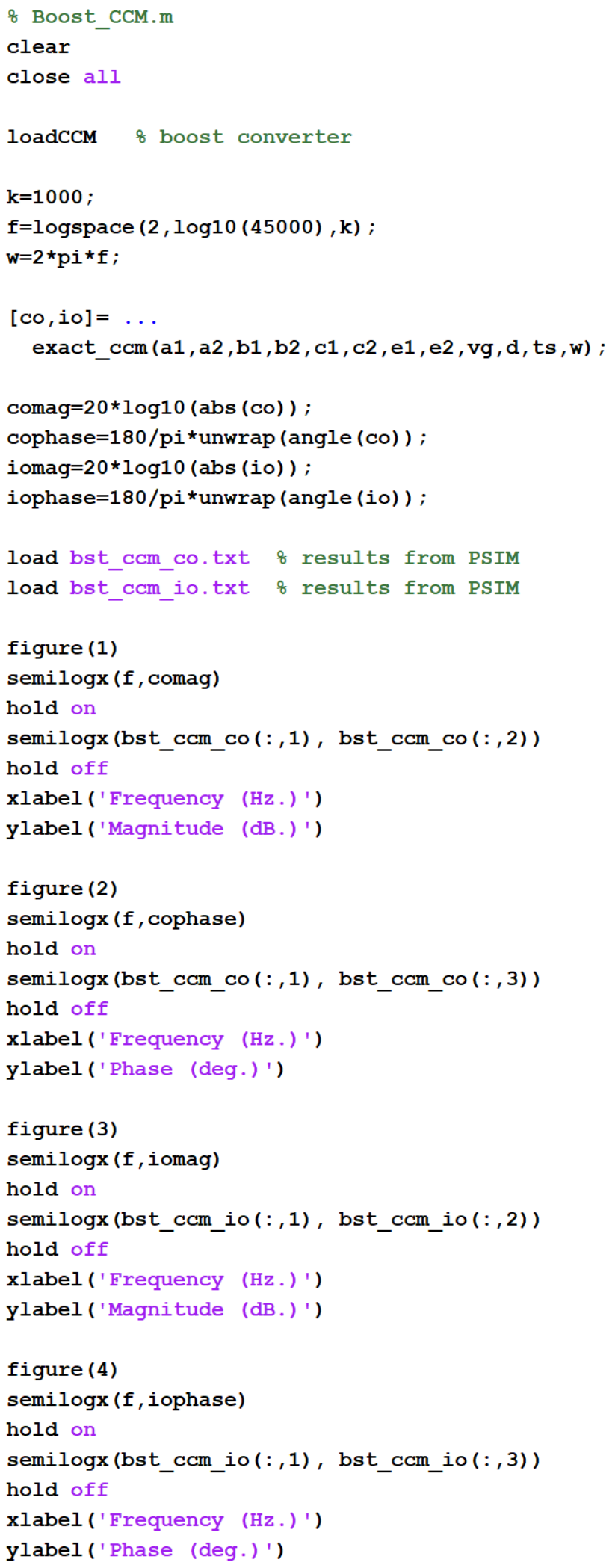

Figure A3. Matlab script: boost_CCM.m. This is the main program which calls the previous functions and plots the frequency responses. 